CARPATHIAN J. MATH.

Volume 38 (2022), No. 1,

Pages 47 - 55
Online version at https : //www . carpathian. cunbm. utcluj. ro/

Print Edition: ISSN 1584 - 2851; Online Edition: ISSN 1843 - 4401

DOI: https://doi.org/10.37193/CJM.2022.01.05

Dedicated to the memory of Academician Mitrofan M. Choban (1942-2021)

\title{
Iterative numerical method for fractional order two-point boundary value problems
}

\author{
Alexandru Mihai Bica and Zoltan Satmari
}

\begin{abstract}
In this paper we develop an iterative numerical method based on Bernstein splines for solving two-point boundary problems associated to differential equations of fractional order $\alpha \in(0,1)$. The convergence of the method is proved by providing the error estimate and it is tested on a numerical example.
\end{abstract}

\section{INTRODUCTION}

The interest for the study of fractional order differential and integral equations is motivated by their applications in fluid dynamics, viscoelasticity (see [4] for the Bagley-Torvik fractional differential model), heat transfer, diffusive transport, signal processing and various areas of engineering, economy, plasma physics, hematopoiesis, epidemiology (see [3], [6], [7], [9] and [14]). A significant development in the fractional calculus, including fractional differential and integral equations, was realized in recent years and the results are presented in the monographs of Baleanu et al. (see [2]), Diethelm (see [6]), Kilbas et al. (see [9]), Lakshmikantham et al. (see [10]), Podlubny (see [16]). Fundamental definitions and results concerning the Caputo fractional derivative can be found in [6]. The construction of the fractional integral equation equivalent with boundary value problems for nonlinear fractional differential equations with Caputo fractional derivative and the corresponding existence results can be found in [1] and the existence of solutions is realized with the Schauder's, Weissinger's and Banach's fixed point theorems (see [1] and [6]). The numerical methods developed for fractional differential equations and for the equivalent integral equation use various techniques such as quadrature based product integration (see [2] and [13]), backward differentiation and Adams type predictor-corrector formulas (see [2]), Adams-Bashforth-Moulton procedures (see [2] and [6]), collocation (see [15]), Runge-Kutta formulas (see [12]), Legendre polynomials (see [8]), shooting method (see [5]), wavelets (see [14]), Bernstein polynomial approximation (see [17]), and variational iteration (see [18]).

Motivated by the above presented potential applications and by the results from [1], in this work we construct an iterative numerical method based on a Bernstein splines approximation procedure involved at each iterative step, for solving the following fractional order boundary value problem

$$
\left\{\begin{array}{c}
D^{\alpha} x(t)=f(t, x(t)), \quad t \in[0, T] \\
a \cdot x(0)+b \cdot x(T)=c
\end{array}\right.
$$

Received: 24.03.2021. In revised form: 28.10.2021. Accepted: 04.11.2021

1991 Mathematics Subject Classification. 65R20.

Key words and phrases. Fractional order differential equations, two-point boundary value problem, iterative numerical method, Bernstein splines.

Corresponding author: Alexandru Mihai Bica; abica@uoradea.ro 
with $\alpha \in(0,1), a, b, c \in \mathbb{R}, a+b \neq 0$ and $f \in C([0, T] \times \mathbb{R})$. For $b=0$, this boundary value problem contains as particular case the initial value problem of fractional order $\alpha \in(0,1)$. The existence and uniqueness of the solution of the boundary value problem (1.1) was investigated in [1] and [6] by using the Banach's fixed point theorem and the numerical solution was obtained in [15] by applying the piecewise polynomial collocation method. In that follows, we establish the uniform boundedness of the sequence of successive approximations associated to the fixed point operator and develop the Bernstein splines iterative method for solving this boundary value problem. The paper is organized as follows: in Section 2 we investigate the integral equation equivalent to the boundary value problem (1.1) proving the uniform boundedness of the corresponding sequence of successive approximations and presenting the iterative numerical method based on Bernstein splines for the solution of (1.1). Section 3 is devoted to the convergence analysis including the discrete and continuous error estimates. In the last section we present two numerical experiments that illustrates the accuracy and the performances of the proposed method and point out some concluding remarks.

\section{THE ITERATIVE NUMERICAL METHOD}

According to Lemma 3.2 in [1] and Lemma 6.40 from [6], the boundary value problem (1.1) is equivalent with the following Volterra-Fredholm integral equation

$$
\begin{aligned}
x(t)=\frac{c}{a+b} & +\frac{1}{\Gamma(\alpha)} \int_{0}^{t}(t-s)^{\alpha-1} f(s, x(s)) d s- \\
& -\frac{b}{(a+b) \Gamma(\alpha)} \int_{0}^{T}(T-s)^{\alpha-1} f(s, x(s)) d s .
\end{aligned}
$$

The existence and uniqueness of the solution of (1.1) in the Banach space $\left(C[0, T],\|\cdot\|_{\infty}\right)$ is proved in Theorem 6.41 from [6], where $C[0, T]=\{f:[0, T] \rightarrow \mathbb{R}, f$ continuous on $[0, T]\}$ and $\|f\|_{\infty}=\max \{|f(t)|: t \in[0, T]\}$.

Theorem 2.1. (Theorem 6.41 in [6]) If $a+b \neq 0, f \in C([0, T] \times \mathbb{R})$, and if $f$ satisfies $a$ Lipschitz condition with Lipschitz constant $L$ with respect to its second argument, then under the condition $L T^{\alpha}\left(1+\frac{|b|}{|a+b|}\right)<\Gamma(\alpha+1)$, the boundary value problem (1.1) has unique solution $x^{*} \in C[0, T]$.

The proof uses the Banach's fixed point theorem applied to the integral operator $A$ : $C[0, T] \rightarrow C[0, T]$, given by

$$
\begin{aligned}
A(x(t))=\frac{c}{a+b} & +\frac{1}{\Gamma(\alpha)} \int_{0}^{t}(t-s)^{\alpha-1} f(s, x(s)) d s- \\
& -\frac{b}{(a+b) \Gamma(\alpha)} \int_{0}^{T}(T-s)^{\alpha-1} f(s, x(s)) d s, \quad t \in[0, T] .
\end{aligned}
$$

This operator is a contraction with the constant $\gamma=\frac{L T^{\alpha}\left(1+\frac{|b|}{|a+b|}\right)}{\Gamma(\alpha+1)}<1$, and considering the sequence of successive approximations associated to this integral operator, $x_{m+1}=$ $A\left(x_{m}\right), m \in \mathbb{N}$, by the Banach's fixed point theorem we get the following estimates:

$$
\left|x^{*}(t)-x_{m}(t)\right| \leq \frac{\gamma^{m}}{1-\gamma} \cdot\left|x_{1}(t)-x_{0}(t)\right|
$$


and

$$
\left|x^{*}(t)-x_{m}(t)\right| \leq \frac{\gamma}{1-\gamma} \cdot\left|x_{m}(t)-x_{m-1}(t)\right|, \forall m \in \mathbb{N}^{*}, \forall t \in[0, T] .
$$

Proposition 2.1. Under the conditions of Theorem 2.1 the sequence of successive approximations is uniformly bounded.

Proof. Taking $x_{0}(t)=\frac{c}{a+b}$, the sequence of successive approximations can be written as

$$
\begin{aligned}
x_{m}(t)=\frac{c}{a+b} & +\frac{1}{\Gamma(\alpha)} \int_{0}^{t}(t-s)^{\alpha-1} f\left(s, x_{m-1}(s)\right) d s- \\
& -\frac{b}{(a+b) \Gamma(\alpha)} \int_{0}^{T}(T-s)^{\alpha-1} f\left(s, x_{m-1}(s)\right) d s, \quad t \in[0, T], m \in \mathbb{N}^{*}
\end{aligned}
$$

and since the operator $A$ is a contraction, we have

$$
\left|x_{m}(t)-x_{m-1}(t)\right|=\left|A\left(x_{m-1}(t)\right)-A\left(x_{m-2}(t)\right)\right| \leq \gamma\left|x_{m-1}(t)-x_{m-2}(t)\right|, \forall t \in[0, T],
$$

and by induction we get

$$
\left|x_{m}(t)-x_{m-1}(t)\right| \leq \gamma^{m-1}\left|x_{1}(t)-x_{0}(t)\right| \leq \frac{M_{0} T^{\alpha}\left(1+\frac{|b|}{|a+b|}\right)}{\Gamma(\alpha+1)}, \forall t \in[0, T]
$$

for all $m \in \mathbb{N}^{*}$, where $M_{0} \geq 0$ is such that $\left|f\left(t, x_{0}(t)\right)\right| \leq M_{0}, \forall t \in[0, T]$. Consequently,

$$
\begin{aligned}
\left|x_{m}(t)\right| & \leq\left|x_{m}(t)-x_{0}(t)\right|+\left|x_{0}(t)\right| \leq \\
& \leq\left(1+\gamma+\ldots+\gamma^{m-1}\right) \cdot \frac{M_{0} T^{\alpha}\left(1+\frac{|b|}{|a+b|}\right)}{\Gamma(\alpha+1)}+\frac{|c|}{|a+b|} \leq R
\end{aligned}
$$

for all $t \in[0, T]$ and $m \in \mathbb{N}^{*}$, that is the uniform boundedness of the sequence $\left(x_{m}\right)_{m \in \mathbb{N}^{*}}$, where $R=\frac{M_{0} T^{\alpha}\left(1+\frac{|b|}{|a+b|}\right)}{\Gamma(\alpha+1)(1-\gamma)}+\frac{|c|}{|a+b|}$. Denoting $F_{m}(t)=f\left(t, x_{m}(t)\right)$ for $t \in[0, T]$ and $m \in \mathbb{N}$, and using the Lipschitz property it obtains,

$$
\left|F_{m}(t)\right| \leq\left|f\left(t, x_{m}(t)\right)-f\left(t, x_{0}(t)\right)\right|+\left|f\left(t, x_{0}(t)\right)\right| \leq \frac{L M_{0} T^{\alpha}\left(1+\frac{|b|}{|a+b|}\right)}{\Gamma(\alpha+1)(1-\gamma)}+M_{0}=M
$$

for all $t \in[0, T]$ and $m \in \mathbb{N}^{*}$, and thus the sequence $\left(F_{m}\right)_{m \in \mathbb{N}}$ is uniformly bounded, too.

In order to construct the iterative method consider a uniform mesh of the interval $[0, T]$ with the knots $t_{i}=i \cdot h, i=\overline{0, n}$, where $h=\frac{T}{n}$ is the stepsize. For each $m \in \mathbb{N}$, and on each subinterval $\left[t_{i-1}, t_{i}\right], i=\overline{1, n}$, we approximate the continuous function $F_{m}$ by the Bernstein polynomial of a given degree $q \geq 1$ :

$$
B_{m, i}(t)=\frac{1}{h^{q}} \sum_{k=0}^{q} C_{q}^{k}\left(t-t_{i-1}\right)^{k}\left(t_{i}-t\right)^{q-k} \cdot F_{m}\left(t_{i-1}+\frac{k h}{q}\right), t \in\left[t_{i-1}, t_{i}\right]
$$

where $C_{q}^{k}=\frac{q !}{k ! \cdot(q-k) !}$. By the inequality of Lorentz (see [11]), in the Bernstein uniform approximation formula $F_{m}(t)=B_{m, i}(t)+R_{m, i}(t)$, we obtain the estimate in terms of the modulus of continuity

$$
\left|R_{m, i}(t)\right| \leq \frac{5}{4} \cdot \omega\left(F_{m}, \frac{h}{\sqrt{q}}\right), \forall t \in\left[t_{i-1}, t_{i}\right], \forall i=\overline{1, n}, \forall m \in \mathbb{N} .
$$


Now, based on (2.5) and (2.7), for $m \in \mathbb{N}^{*}=\mathbb{N} \backslash\{0\}$ we get the following iterative form of (2.5) on the knots:

$$
\begin{aligned}
x_{m}\left(t_{0}\right) & =\frac{c}{a+b}-\frac{b}{(a+b) \Gamma(\alpha)} \sum_{i=1}^{n} \int_{t_{i-1}}^{t_{i}}(T-s)^{\alpha-1}\left(B_{m-1, i}(s)+R_{m-1, i}(s)\right) d s \\
x_{m}\left(t_{k}\right) & =\frac{c}{a+b}+\frac{1}{\Gamma(\alpha)} \sum_{i=1}^{k} \int_{t_{i-1}}^{t_{i}}\left(t_{k}-s\right)^{\alpha-1}\left(B_{m-1, i}(s)+R_{m-1, i}(s)\right) d s- \\
& -\frac{b}{(a+b) \Gamma(\alpha)} \sum_{i=1}^{n} \int_{t_{i-1}}^{t_{i}}(T-s)^{\alpha-1}\left(B_{m-1, i}(s)+R_{m-1, i}(s)\right) d s, \quad k=\overline{1, n} .
\end{aligned}
$$

If we compute the integrals from (2.10) by using the Bernstein polynomial formula (2.7) and the change variable $s=t_{i-1}+u h$, we obtain

$$
\int_{t_{i-1}}^{t_{i}}\left(s-t_{i-1}\right)^{j}\left(t_{i}-s\right)^{q-j}\left(t_{k}-s\right)^{\alpha-1} d s=h^{q+\alpha} \cdot \psi_{j, k}(i)
$$

where $\psi_{j, k}(i)=\int_{0}^{1} u^{j}(1-u)^{q-j}(k-i-u+1)^{\alpha-1} d u$. In this way, for $m \in \mathbb{N}^{*}$, considering $x_{0}(t)=\frac{c}{a+b}, \forall t \in[0, T]$, it obtains the following iterative algorithm:

$$
\begin{aligned}
x_{m}\left(t_{0}\right)=\frac{c}{a+b} & -\frac{b h^{\alpha}}{(a+b) \Gamma(\alpha)} \sum_{i=1}^{n} \sum_{j=0}^{q} C_{q}^{j} \psi_{j, n}(i) \overline{F_{m-1, i}}\left(t_{i-1}+\frac{j h}{q}\right)+\overline{R_{m, 0}} \\
x_{m}\left(t_{k}+\frac{l h}{q}\right)= & \frac{c}{a+b}+\frac{h^{\alpha}}{\Gamma(\alpha)}\left[\sum_{i=1}^{k} \sum_{j=0}^{q} C_{q}^{j} \psi_{j, k+\frac{l}{q}}(\nu) \overline{F_{m-1, i}}\left(t_{i-1}+\frac{j h}{q}\right)+\right. \\
& \left.+\sum_{j=0}^{q} C_{q}^{j} \cdot \psi_{j, k+\frac{l}{q}}\left(\nu^{\prime}\right) \overline{F_{m-1, k}}\left(t_{k}+\frac{j h}{q}\right)\right]- \\
& -\frac{b h^{\alpha}}{(a+b) \Gamma(\alpha)} \sum_{i=1}^{n} \sum_{j=0}^{q} C_{q}^{j} \psi_{j, n}(i) \overline{F_{m-1, i}}\left(t_{i-1}+\frac{j h}{q}\right)+\overline{R_{m, k+\frac{l}{q}}}
\end{aligned}
$$

for $k=\overline{0, n-1}, l=\overline{0, q}$, where $\psi_{j, k+\frac{l}{q}}(\nu)=\int_{0}^{1} u^{j}(1-u)^{q-j}(\nu-u)^{\alpha-1} d u$, with $\nu=$ $k+\frac{l}{q}-(i-1)$ and

$$
\psi_{j, k+\frac{l}{q}}\left(\nu^{\prime}\right)=\int_{0}^{1} u^{j}(1-u)^{q-j}\left(\frac{l}{q}-u\right)^{\alpha-1} d u .
$$

Here, we have denoted $\overline{F_{m-1, i}}\left(t_{i-1}+\frac{j h}{q}\right)=f\left(t_{i-1}+\frac{j h}{q}, \overline{x_{m-1}}\left(t_{i-1}+\frac{j h}{q}\right)\right)$ and the first sum in (2.12) appears only for $k \geq 1$. The expressions (2.11)-(2.12) can be written in the form $x_{m}\left(t_{k}+\frac{l h}{q}\right)=\overline{x_{m}}\left(t_{k}+\frac{l h}{q}\right)+\overline{R_{m, k+\frac{l}{q}}}$, with $k=\overline{0, n-1}, l=\overline{0, q}$. The algorithm has the following stopping criterion: with given $\varepsilon>0$ find the first natural number $m \in \mathbb{N}^{*}$ for which $\left|\overline{x_{m}}\left(t_{k}\right)-\overline{x_{m-1}}\left(t_{k}\right)\right|<\varepsilon, \forall k=\overline{0, n}$, and stop at this iteration " $m$ ". 
The iterative method of Bernstein splines provides a continuous approximation of the solution, too. More precisely, by using the values $\overline{x_{m}}\left(t_{k}+\frac{l h}{q}\right), k=\overline{0, n-1}, l=\overline{0, q}$, computed at the last iterative step, we construct the Bernstein spline $\overline{B_{m, q}}:[0, T] \rightarrow \mathbb{R}$, that on each subinterval $\left[t_{i-1}, t_{i}\right], i=\overline{1, n}$, has the expression

$$
\overline{B_{m, q}}(t)=\frac{1}{h^{q}} \sum_{j=0}^{q} C_{q}^{j}\left(t-t_{i-1}\right)^{j}\left(t_{i}-t\right)^{q-j} \cdot \overline{x_{m}}\left(t_{i-1}+\frac{j h}{q}\right), t \in\left[t_{i-1}, t_{i}\right] .
$$

\section{CONVERGENCE ANALYSIS}

Now we can state our main result.

Theorem 3.2. Under the conditions of Theorem 2.1, the terms $\overline{x_{m}}\left(t_{k}\right), k=\overline{0, n}, m \in \mathbb{N}^{*}$ computed in (2.11)-(2.12) approximate the solution of the boundary value problem (1.1) on the mesh knots with the error estimate,

$$
\begin{aligned}
\left|x^{*}\left(t_{k}\right)-\overline{x_{m}}\left(t_{k}\right)\right| & \leq \frac{M_{0} T^{\alpha}\left(1+\frac{|b|}{|a+b|}\right) \gamma^{m}}{(1-\gamma) \Gamma(\alpha+1)}+ \\
& +\frac{5 T^{\alpha}\left(1+\frac{|b|}{|a+b|}\right) \omega\left(F_{m-1}, \frac{h}{\sqrt{q}}\right)}{4(1-\gamma) \Gamma(\alpha+1)}, \forall k=\overline{0, n}, m \in \mathbb{N}^{*} .
\end{aligned}
$$

Moreover, the error estimate in the continuous approximation of the solution is:

$$
\begin{aligned}
\left|x^{*}(t)-\overline{B_{m, q}}(t)\right| & \leq \frac{\gamma^{m}}{1-\gamma} \cdot \frac{M_{0} T^{\alpha}\left(1+\frac{|b|}{|a+b|}\right)}{\Gamma(\alpha+1)}+ \\
& +\frac{5 T^{\alpha}\left(1+\frac{|b|}{|a+b|}\right) \omega\left(F_{m-1}, \frac{h}{\sqrt{q}}\right)}{4(1-\gamma) \Gamma(\alpha+1)}+\frac{5}{4} \omega\left(V_{m}, \frac{h}{\sqrt{q}}\right), \forall t \in[0, T], m \in \mathbb{N}^{*}
\end{aligned}
$$

where $V_{m}$ is given in (3.18).

Proof. In inductive manner, by (2.9)-(2.10) we get

$$
\begin{aligned}
x_{1}\left(t_{0}\right) & =\frac{c}{a+b}-\frac{b}{(a+b) \Gamma(\alpha)} \sum_{i=1}^{n} \sum_{j=0}^{q} C_{q}^{j} h^{\alpha} \psi_{j, n}(i) F_{0}\left(t_{i-1}+\frac{j h}{q}\right)- \\
& -\frac{b}{(a+b) \Gamma(\alpha)} \sum_{i=1}^{n} \int_{t_{i-1}}^{t_{i}}(T-s)^{\alpha-1} R_{0, i}(s) d s=\overline{x_{1}}\left(t_{0}\right)+R_{1,0}
\end{aligned}
$$

with $\left|R_{1,0}\right| \leq \frac{5|b| T^{\alpha}}{4|a+b| \Gamma(\alpha+1)} \cdot \omega\left(F_{0}, \frac{h}{\sqrt{q}}\right)$, and

$$
\begin{aligned}
x_{1}\left(t_{k}\right)= & \frac{c}{a+b}+\frac{1}{\Gamma(\alpha)} \sum_{i=1}^{k} \int_{t_{i-1}}^{t_{i}}\left(t_{k}-s\right)^{\alpha-1}\left(B_{0, i}(s)+R_{0, i}(s)\right) d s- \\
& -\frac{b}{(a+b) \Gamma(\alpha)} \sum_{i=1}^{n} \int_{t_{i-1}}^{t_{i}}(T-s)^{\alpha-1}\left(B_{0, i}(s)+R_{0, i}(s)\right) d s \\
= & \overline{x_{1}}\left(t_{k}\right)+\overline{R_{1}}\left(t_{k}\right), k=\overline{1, n},
\end{aligned}
$$


with $\left|\overline{R_{1}}\left(t_{k}\right)\right| \leq \frac{5 T^{\alpha}}{4 \Gamma(\alpha+1)}\left(1+\frac{|b|}{|a+b|}\right) \cdot \omega\left(F_{0}, \frac{h}{\sqrt{q}}\right)$. Consequently, by using the change variable $s-t_{i-1}=u h$, we have

$$
\begin{gathered}
\sum_{i=1}^{k} \int_{t_{i-1}}^{t_{i}}\left[\sum_{j=0}^{q} C_{q}^{j}\left(s-t_{i-1}\right)^{j}\left(t_{i}-s\right)^{q-j}\right]\left(t_{k}-s\right)^{\alpha-1} d s= \\
=h^{q+\alpha} \sum_{i=1}^{k} \int_{0}^{1}\left[\sum_{j=0}^{q} C_{q}^{j} u^{j}(1-u)^{q-j}\right](k-i+1-u)^{\alpha-1} d u \leq \frac{T^{\alpha} h^{q}}{\alpha} .
\end{gathered}
$$

and since for $k=\overline{1, n}$ :

$$
\begin{aligned}
& x_{2}\left(t_{k}\right)=\frac{c}{a+b}+ \\
& +\frac{1}{\Gamma(\alpha)} \sum_{i=1}^{k} \int_{t_{i-1}}^{t_{i}} \frac{1}{h^{q}} \sum_{j=0}^{q} C_{q}^{j}\left(s-t_{i-1}\right)^{j}\left(t_{i}-s\right)^{q-j} \overline{F_{1}}\left(t_{i-1}+\frac{j h}{q}\right) \cdot\left(t_{k}-s\right)^{\alpha-1} d s+ \\
& +\frac{1}{\Gamma(\alpha)} \sum_{i=1}^{k} \int_{t_{i-1}}^{t_{i}} \frac{1}{h^{q}} \sum_{j=0}^{q} C_{q}^{j}\left(s-t_{i-1}\right)^{j}\left(t_{i}-s\right)^{q-j} \overline{R_{1}}\left(t_{i-1}+\frac{j h}{q}\right)\left(t_{k}-s\right)^{\alpha-1} d s- \\
& -\frac{b}{(a+b) \Gamma(\alpha)} \sum_{i=1}^{n} \int_{t_{i-1}}^{t_{i}} \frac{1}{h^{q}} \sum_{j=0}^{q} C_{q}^{j}\left(s-t_{i-1}\right)^{j}\left(t_{i}-s\right)^{q-j} \overline{F_{1}}\left(t_{i-1}+\frac{j h}{q}\right) \cdot\left(t_{n}-s\right)^{\alpha-1} d s- \\
& -\frac{b}{(a+b) \Gamma(\alpha)} \sum_{i=1}^{n} \int_{t_{i-1}}^{t_{i}} \frac{1}{h^{q}} \sum_{j=0}^{q} C_{q}^{j}\left(s-t_{i-1}\right)^{j}\left(t_{i}-s\right)^{q-j} \overline{R_{1}}\left(t_{i-1}+\frac{j h}{q}\right) \cdot\left(t_{n}-s\right)^{\alpha-1} d s \\
& =\overline{x_{2}}\left(t_{k}\right)+\overline{R_{2}}\left(t_{k}\right)
\end{aligned}
$$

it obtains

$$
\left|\overline{R_{2}}\left(t_{k}\right)\right| \leq\left[1+\frac{L T^{\alpha}\left(1+\frac{|b|}{|a+b|}\right)}{\Gamma(\alpha+1)}\right] \cdot \frac{5 T^{\alpha}}{4 \Gamma(\alpha+1)}\left(1+\frac{|b|}{|a+b|}\right) \omega\left(F_{1}, \frac{h}{\sqrt{q}}\right)
$$

and similarly, $\left|\overline{R_{2}}\left(t_{0}\right)\right| \leq\left[1+\frac{|b| L T^{\alpha}}{|a+b| \Gamma(\alpha+1)}\right] \cdot \frac{5|b| T^{\alpha}}{4|a+b| \Gamma(\alpha+1)} \omega\left(F_{1}, \frac{h}{\sqrt{q}}\right)$. By induction, for $m \geq 2$, we get

$$
\begin{aligned}
\left|\overline{R_{m, 0}}\right| \leq[1+ & \left.\frac{|b| L T^{\alpha}}{|a+b| \Gamma(\alpha+1)}+\cdots+\left(\frac{|b| L T^{\alpha}}{|a+b| \Gamma(\alpha+1)}\right)^{m-1}\right] \\
& \cdot \frac{5|b| T^{\alpha}}{4|a+b| \Gamma(\alpha+1)} \cdot \omega\left(F_{m-1}, \frac{h}{\sqrt{q}}\right)
\end{aligned}
$$

and

$$
\begin{aligned}
\left|\overline{R_{m}}\left(t_{k}\right)\right| \leq\left[1+\gamma+\cdots+\gamma^{m-1}\right] & \\
& \cdot \frac{5 T^{\alpha}}{4 \Gamma(\alpha+1)}\left(1+\frac{|b|}{|a+b|}\right) \omega\left(F_{m-1}, \frac{h}{\sqrt{q}}\right), k=\overline{1, n} .
\end{aligned}
$$

Considering $\left|x^{*}\left(t_{k}\right)-\overline{x_{m}}\left(t_{k}\right)\right| \leq\left|x^{*}\left(t_{k}\right)-x_{m}\left(t_{k}\right)\right|+\left|x_{m}\left(t_{k}\right)-\overline{x_{m}}\left(t_{k}\right)\right|$, by (3.16)-(3.17) and according to the estimates (2.3)-(2.6), the inequality (3.14) follows. Now we define the 
auxiliary function $V_{m}:[0, T] \rightarrow \mathbb{R}$ which on each subinterval $\left[t_{i}, t_{i+1}\right], i=\overline{0, n-1}$ has the expression

$$
V_{m}(t)=x_{m}(t)+\frac{t_{i+1}-t}{h}\left(\overline{x_{m}}\left(t_{i}\right)-x_{m}\left(t_{i}\right)\right)+\frac{t-t_{i}}{h}\left(\overline{x_{m}}\left(t_{i+1}\right)-x_{m}\left(t_{i+1}\right)\right) .
$$

We see that $V_{m}$ is continuous on $[0, T]$ with $V_{m}\left(t_{i}\right)=\overline{x_{m}\left(t_{i}\right)}, \forall i=\overline{0, m}$, and thus $\overline{B_{m, q}}$ interpolates $V_{m}$ on the mesh knots, having $\left|V_{m}(t)-\overline{B_{m, q}}(t)\right| \leq \frac{5}{4} \omega\left(V_{m}, \frac{h}{\sqrt{q}}\right)$ for all $t \in$ $\left[t_{i}, t_{i+1}\right], i=\overline{0, n-1}$. Then, because

$$
\left|x_{m}(t)-V_{m}(t)\right| \leq \max _{i=\overline{0, n-1}}\left\{\left|\overline{x_{m}}\left(t_{i}\right)-x_{m}\left(t_{i}\right)\right|,\left|\overline{x_{m}}\left(t_{i+1}\right)-x_{m}\left(t_{i+1}\right)\right|\right\}
$$

from the inequality

$$
\left|x^{*}(t)-\overline{B_{m, q}}(t)\right| \leq\left|x^{*}(t)-x_{m}(t)\right|+\left|x_{m}(t)-V_{m}(t)\right|+\left|V_{m}(t)-\overline{B_{m, q}}(t)\right|
$$

we obtain the estimate (3.15). By the continuity of the functions $F_{m}$ and $V_{m}$, for $m \in \mathbb{N}^{*}$, we infer that $\lim _{h \rightarrow 0} \omega\left(F_{m-1}, \frac{h}{\sqrt{q}}\right)=\lim _{h \rightarrow 0} \omega\left(V_{m}, \frac{h}{\sqrt{q}}\right)=0$, and since $\gamma \in(0,1)$, by (3.14) and (3.15) the convergence of the iterative method follows.

\section{NUMERICAL EXPERIMENTS}

In order to test the convergence theoretical result stated in Theorem 3.2 and to illustrate the accuracy of the iterative method we consider the following numerical examples.

Example 1. The boundary value problem

$$
\left\{\begin{array}{l}
D^{0.5} x(t)=\frac{4 \sqrt{t}}{\Gamma\left(\frac{3}{2}\right)}+2 t \sqrt{t}-\sqrt{t} \cdot x(t), \quad t \in[0,1] \\
x(0)+x(1)=2
\end{array}\right.
$$

has the exact solution $x^{*}(t)=2 t$ (see [15]). Applying the iterative algorithm with $q=$ 1 and $q=4$, and considering $m=10$ iterations, in Table 1 we represent the obtained numerical results for $e_{n, i}=\left|\overline{x_{m}}\left(t_{i}\right)-x^{*}\left(t_{i}\right)\right|, i=\overline{0, n}$. The convergence was tested taking $n=20, n=50$, and $n=100$. Investigating the columns of Table 1 , we see that the errors decrease by passing through $n=20, n=50, n=100$ in both cases $q=1$ and $q=4$, and in addition, the results for $q=4$ are better. Denoting $\sigma=\max _{i=\overline{0, n}} e_{n, i}$, the values of $\sigma$ are represented in the first line of Table 1. In order to compare the values of $\sigma$ with the similar results $\varepsilon_{N}=\max _{i=\overline{0, N}}\left|x_{N}\left(t_{i}\right)-x^{*}\left(t_{i}\right)\right|$ obtained in [15], Table 5.1 for uniform partitions (which means $r=1$, in the context of graded mesh), we have specified in the last two lines of Table 1 the corresponding values of $\varepsilon_{N}$ for $\mathrm{N}=32, \mathrm{~N}=64$ and $\mathrm{N}=128$, respectively. 


\begin{tabular}{|l|l|l|l|l|l|l|}
\hline$q:$ & \multicolumn{3}{|c|}{1} & \multicolumn{3}{c|}{4} \\
\hline$\sigma:$ & $8,06 E-03$ & $2,03 E-03$ & $7,53 E-04$ & $2,38 E-03$ & $6,47 E-04$ & $2,70 E-04$ \\
\hline$t_{i}$ & $n=20$ & $n=50$ & $n=100$ & $n=20$ & $n=50$ & $n=100$ \\
\hline 0,0 & $7,26 E-04$ & $1,41 E-04$ & $1,35 E-05$ & $1,77 E-04$ & $2,95 E-06$ & $3,51 E-05$ \\
\hline 0,1 & $8,06 E-03$ & $2,03 E-03$ & $7,53 E-04$ & $2,38 E-03$ & $6,47 E-04$ & $2,70 E-04$ \\
\hline 0,2 & $4,83 E-03$ & $1,26 E-03$ & $4,89 E-04$ & $1,47 E-03$ & $4,25 E-04$ & $1,96 E-04$ \\
\hline 0,3 & $3,44 E-03$ & $9,14 E-04$ & $3,68 E-04$ & $1,07 E-03$ & $3,23 E-04$ & $1,60 E-04$ \\
\hline 0,4 & $2,61 E-03$ & $7,02 E-04$ & $2,88 E-04$ & $8,18 E-04$ & $2,54 E-04$ & $1,31 E-04$ \\
\hline 0,5 & $2,05 E-03$ & $5,48 E-04$ & $2,23 E-04$ & $6,40 E-04$ & $1,96 E-04$ & $9,87 E-05$ \\
\hline 0,6 & $1,63 E-03$ & $4,25 E-04$ & $1,62 E-04$ & $4,98 E-04$ & $1,40 E-04$ & $6,22 E-05$ \\
\hline 0,7 & $1,31 E-03$ & $3,21 E-04$ & $1,06 E-04$ & $3,82 E-04$ & $8,79 E-05$ & $2,37 E-05$ \\
\hline 0,8 & $1,06 E-03$ & $2,37 E-04$ & $5,80 E-05$ & $2,87 E-04$ & $4,31 E-05$ & $1,03 E-05$ \\
\hline 0,9 & $8,67 E-04$ & $1,76 E-04$ & $2,58 E-05$ & $2,19 E-04$ & $1,32 E-05$ & $3,17 E-05$ \\
\hline 1,0 & $7,26 E-04$ & $1,41 E-04$ & $1,35 E-05$ & $1,77 E-04$ & $2,95 E-06$ & $3,51 E-05$ \\
\hline$\varepsilon_{N}$ & $5,14 E-03$ & $2,59 E-03$ & $1,30 E-03$ & $5,14 E-03$ & $2,59 E-03$ & $1,30 E-03$ \\
\hline $\mathrm{N}:$ & $N=32$ & $N=64$ & $N=128$ & $N=32$ & $N=64$ & $N=128$ \\
\hline
\end{tabular}

Table 1. The numerical results for the cases $q=1$ and $q=4$, versus the results in [15]

Comparing the results provided by the Bernstein splines method with those obtained in [15] (last 2 rows in the table), we see that the Bernstein splines method gives better results and the order of accuracy is improved for $n=50$ and $n=100$, when $q=4$ is chosen.

Example 2. The exact solution of the following boundary value problem involving a nonlinear differential equation:

$$
\left\{\begin{array}{l}
D^{0.5} x(t)=\frac{\sqrt{t}}{\Gamma\left(\frac{3}{2}\right)}+\frac{1}{4} \cdot[x(t)]^{2}-\frac{t^{2}}{4}, \quad t \in[0,1] \\
x(0)+x(1)=1
\end{array}\right.
$$

is $x^{*}(t)=t$ and applying the iterative algorithm with $q=1$ and $q=4$, after $m=10$ iterations we obtain the numerical results expressed for $e_{n, i}=\left|\overline{x_{m}}\left(t_{i}\right)-x^{*}\left(t_{i}\right)\right|, i=\overline{0, n}$ in Table 2. Here, $\max =\max _{i=\overline{0, n}}\left|\overline{x_{m}}\left(t_{i}\right)-x^{*}\left(t_{i}\right)\right|$, and investigating the results from Table 2 we infer that the convergence of the iterative method is confirmed and taking $q=4$ the results are improved again, similarly to the case of the first linear example.

\begin{tabular}{|l|l|l|l|l|l|l|}
\hline$q:$ & \multicolumn{3}{|c|}{1} & \multicolumn{3}{c|}{4} \\
\hline$t_{i}$ & $n=20$ & $n=50$ & $n=100$ & $n=20$ & $n=50$ & $n=100$ \\
\hline 0,2 & $2,49 E-03$ & $6,24 E-04$ & $2,20 E-04$ & $7,37 E-04$ & $1,86 E-04$ & $6,59 E-05$ \\
\hline 0,5 & $1,37 E-03$ & $3,46 E-04$ & $1,22 E-04$ & $4,09 E-04$ & $1,04 E-04$ & $3,66 E-05$ \\
\hline 0,8 & $1,05 E-03$ & $2,64 E-04$ & $9,34 E-05$ & $3,13 E-04$ & $7,93 E-05$ & $2,80 E-05$ \\
\hline $\max$ & $3,92 E-03$ & $9,64 E-04$ & $3,39 E-04$ & $1,14 E-03$ & $2,87 E-04$ & $1,01 E-04$ \\
\hline
\end{tabular}

Table 2. Numerical results in the cases $q=1$ and $q=4$ for Example 2

\section{CONCLUSION}

In this work we proved that the proposed iterative method of Bernstein splines, suitable for fractional order differential and integral equations, is convergent and effective both for linear and nonlinear equations. The convergence is obtained in Theorem 3.2 providing the error estimate in terms of the modulus of continuity for the discrete and continuous approximation. The presented numerical examples confirm this theoretical result and the results are improved by increasing the degree $q$ of the involved Bernstein 
polynomials. We chosed the degree $q=4$ in order to ensure an equilibrium between the increased accuracy requirement and the accumulating errors due to the complexity of calculus. The choice of using piecewise Bernstein polynomials is based on their nice uniform approximation and shape preserving properties.

\section{REFERENCES}

[1] Agarwal R. P.; Benchohra, M.; Hamani, S. A survey on existence results for boundary value problems of nonlinear fractional differential equations and inclusions. Acta Appl. Math. 109 (2010), 973-1033.

[2] Baleanu, D.; Diethelm, K.; Scalas, E.; Trujillo, J. J. Fractional Calculus: Models and Numerical Methods, second ed., World Scientific Publishers, Co., New Jersey, 2016.

[3] Bachher, M.; Sarkar, N.; Lahiri, A. Fractional order thermoelastic interactions in an infinite porous material due to distributed timedependent heat sources. Meccanica 50 (2015), 2167-2178.

[4] Bagley, R. L.; Calico, R. A. Fractional order state equations for the control of viscoelastically damped structures. J. Guid. Contr. Dynam. 14 (1991), 304-311.

[5] Cen, Z.; Huang, J.; Xu, A. An efficient numerical method for a two-point boundary value problem with a Caputo fractional derivative. J. Comput. Appl. Math. 336 (2018), 1-7.

[6] Diethelm, K. The Analysis of Fractional Differential Equations. Lecture Notes in Mathematics. 2004, Springer-Verlag Berlin Heidelberg, 2010.

[7] Goswami, A.; Sushila, J.; Singh, J.; Kumar, D. Numerical computation of fractional Kersten-Krasil'shchik coupled KdV-mKdV system occurring in multi-component plasmas. AIMS Math. 5 (2020), 2346-2368.

[8] Kawala, A. M. Numerical solution for initial and boundary value problems of fractional order. Adv. Pure Math. 8 (2018), 831-844.

[9] Kilbas, A. A.; Srivastava, H. M.; Trujillo, J. J. Theory and Applications of Fractional Differential Equations. Elsevier Science B. V., Amsterdam 2006.

[10] Lakshmikantham, V.; Leela, S.; Vasundhara, J. Theory of Fractional Dynamic Systems. Cambridge Academic Publishers, Cambridge, 2009.

[11] Lorentz, G. G. Bernstein Polynomials, Toronto, Univ. Toronto Press, 1953.

[12] Lubich, C. Runge-Kutta theory for Volterra and Abel integral equations of the second kind. Math. Comp. 41 (1983), no. 163, 87-102.

[13] Micula, S. An iterative numerical method for fractional integral equations of the second kind. J. Comput. Appl. Math. 339 (2018), 124-133.

[14] Mohammad, M.; Trounev, A., Implicit Riesz wavelets based-method for solving singular fractional integrodifferential equations with applications to hematopoietic stem cell modeling. Chaos Solitons Fractals 138 (2020), 109991.

[15] Pedas, A.; Tamme, E. Piecewise polynomial collocation for linear boundary value problems of fractional differential equations. J. Comput. Appl. Math. 236 (2012), 3349-3359.

[16] Podlubny, I. Fractional Differential Equation, Academic Press, San Diego, 1999

[17] Usta, F. Numerical analysis of fractional Volterra integral equations via Bernstein approximation method. J. Comput. Appl. Math. 384 (2021), 113198.

[18] Wu, G. C.; Baleanu, D. Variational iteration method for fractional calculus - a universal approach by Laplace transform. Adv. Differential Equations 2013 (2013), 1-9.

DEPARTMENT OF MATHEMATICS AND INFORMATICS

UNIVERSITY OF ORADEA

UNIVERSITĂŢII 1, 410087, ORADEA, ROMANIA

Email address: abica@uoradea.ro

Email address: zsatmari@yahoo.com 